\title{
Research on Ring Fire Diagnosis of DC Motor Based on Discrete Wavelet Lipschitz Index
}

\author{
Tang Zhao-ping ${ }^{1, *}$, Yang Qing-ping ${ }^{1}$, Tang Shuai ${ }^{2}$, Zhang Wen-sheng ${ }^{2}$ and Sun Jian-ping ${ }^{3}$ \\ ${ }^{1}$ School of Information Engineering, East China Jiao Tong University, Nanchang 330013, China \\ ${ }^{2}$ School of Mechanical and Electrical Engineering, East China Jiao Tong University, Nanchang Jiangxi, 330013, China \\ ${ }^{3}$ School of Railway Transportation, East China Jiao Tong University, Nanchang Jiangxi, 330013, China
}

\begin{abstract}
The favorable localization features of discrete wavelet provide a new method for detecting the mutational points of electric spark signal. In this paper, by means of discrete wavelet function called db5, using the way of 6 scales wavelet, analyzing the gathered electric spark signal and by extracting the modulus maxima of the 6 layers detailed signal coefficient, the signal's mutational points were located exactly and successfully. In addition, via the modulus maxima to calculate Lipschitz index, measuring signal's singularity, the signal's mutational time was confirmed. The result of the simulation shows that this method can detect not only the time and size effectively if the ring fire happens but also the failure of the locomotive traction dc motor, timely and precisely. In this way, the operation safety of the train is ensured.
\end{abstract}

Keywords: Motor ring fire, discrete wavelet, modulus maxima, Lipschitz index.

\section{INTRODUCTION}

Dc motor is universally used in locomotive traction motor due to its good performance of start-up and speed adjustment. However, due to the inherent defect of the dc motor commutator, a large spark can occur and in turn damage the motor when locomotive traction motor is in operation with high power and high current, especially during the harsh weather. This is also called motor ring fire failure [1]. The intense arc can burn the surface of the commutator and the electric brush. The working state of motor is changed from electromotor to the electric generator and the electricity passes through the arc directly into the excitation group, which results in tremendous excitation electricity and a giant back electromotive force. This leads to a huge reversecurrent, produced in armature winding, but conversely burns it out, and causes friction fault and large braking torque, preventing the normal operation of the train. All of these factors give rise to enormous economic losses to the railway transportation services. As a result, particular importance should be given to the early detection of motor ring fire status.

Nowadays, collection of spark signal data is of utmost importance for detecting the motor ring fire and post processing method is mainly divided into two categories:

1. Detecting the extent of the ring fire by analyzing the electric signal converted from the spark signal.

2. The research on spectrum of spark signal.

*Address correspondence to this author at the Shuanggang Road, Nanchang, China. Postcard: 330013; Tel: (0086) 791-87046242;

E-mail: tzp@ecjtu.jx.cn
Spectrum analysis is a little difficult due to the weak spark in the commutator when the motor ring fire occurs. Therefore, in a majority of existing detection methods on ring fire are turning the spark signal to the electric signal and then analyzing the electric signal.

This paper studies the wavelet analysis of the spark signal collected by sensors, and then calculates the index of Lip by figuring out the modulus maxima of the coefficient matrix selected from the all low-frequency signal layers. Thereby, the ring fire failure of locomotive traction motor can be easily detected.

\section{PROCESSING PRINCIPLE OF MODULUS MAXI- MA BASED ON WAVELET ANALYSIS}

\subsection{Analysis Theory of Discrete Wavelet}

Wavelet analysis has excellent localization performance both in time domain and frequency domain, so different scales are adopted for signal observation. The wavelet analysis method, which has favorable localization features, is particularly suitable for feature extraction of nonstationary random signal. Since the motor spark signal belongs to the transient, non-stationary random signal and rendering simulated spark signal into digital signal conducted by $\mathrm{A} / \mathrm{D}$ acquisition card, this paper, as a consequence, applies discrete wavelet transform to conduct the analysis of signal.

Discrete wavelet transform always adopts Mallat algorithm, containing the signal's wavelet decomposition and reconstruction. Mallat algorithm's decomposition formula: 
$C_{k}^{j+1}=\sum_{n \in z} h(n-2 k) C_{n}^{j}$

$D_{k}^{j+1}=\sum_{n \in z} g(n-2 k) C_{n}^{j}$

Mallat algorithm's reconstruction formula:

$C_{n}^{j-1}=\sum_{k \in z} h(n-2 k) C_{k}^{j}+\sum_{k \in z} g(n-2 k) D_{k}^{j} \quad, \quad$ among it, $h(n)=2^{-\frac{1}{2}} \int \varnothing\left(\frac{1}{2} x\right) \varnothing(x-n) d x$, the $h(n)$ includes Normalization factor $2^{-\frac{1}{2}}, \varnothing(x)$ is wavelet scaling function. $g(n)=2^{-\frac{1}{2}} \int \varphi\left(\frac{1}{2} x\right) \varphi(x-n) d x, \varphi(n)$ is wavelet function. $g(n)$ is high-pass filtering function, $h(n)$ is low-pass filtering function. High frequency part represents the signal's detailed information; on the contrary low frequency part denotes the signal's profile message [2].

\subsection{The Choice of Wavelet Function}

The wavelet function in wavelet transform is irregular and non-unique. The processing results will have great difference if the same signal is analyzed by the different wavelet functions. In consequence, the choice of the optimal wavelet function has a great impact on the accuracy of results.

According to the similarity, which is between the low frequency reconstruction signal from the analyzed wavelet signal and the low frequency signal from the original one, the optimum wavelet basis is chosen (Similarity is reflected by the size of feature variance). The more the similarity is, the more thorough the high frequency components of the spark signal decomposition will be [3]. The conclusion, feature variance is minimum when using db5 to conduct 6 layers wavelet decomposition, acquired from the experiments when selecting the different numbers of $\mathrm{db}$ wavelet function and decomposition layer. That is to say, the low frequency reconstruction signal after the wavelet analysis and the low frequency signal from the original one are extremely similar. For this reason, this paper employs 6 scales wavelet decomposition whose wavelet basis is $\mathrm{db} 5$.

\subsection{Signal's Singularity Detection Based on Wavelet Analysis}

A large spark in commutator will be produced if the ring fire occurs in traction motor. And the gathered spark signal is presented through mutation; it is the same as if the spark signal has singularities. In consequence, it is the signal singularity of wavelet analysis that can judge whether the traction motor would generate ring fire failure. To solve the location of signal singular points and to judge the size of the signal singularity are the main task of the wavelet singularity detection.

Supposing $W_{f}(a, b)$ is the wavelet analysis of signal $f(t)$, in $s_{0}$ scale, the point $\left(s_{0}, t_{0}\right)$ is a local extreme point if there is a dot $\left(s_{0}, t_{0}\right)$ that satisfies $\frac{\partial W_{f}\left(s, t_{0}\right)}{\partial t}=0$, in addition, the equation $\frac{\partial W_{f}\left(s_{0}, t_{0}\right)}{\partial t}=0$ has a zero-crossing point at the $t_{0}$ time. The point $\left(s_{0}, t_{0}\right)$ is called the modulus maxim point of wavelet analysis, if $\left|\partial W_{f}\left(s_{0}, t\right)\right|<=\left|\partial W_{f}\left(s_{0}, t_{0}\right)\right|$ is suitable with any point in the neighborhood of $t_{0}$.

\subsubsection{The Signal Singularity Point Positioning of Modu- lus Maxima Based on Wavelet Transform Coefficient}

Grossmann systematically expounded how to use the localization features of wavelet analysis to position the singular points of signal. Arranging a smoothing function $\theta(t)$ meets the conditions: $\theta(t)=0\left(1 /\left(1+t^{2}\right)\right)$ and $\int_{R} \theta(t) d t \neq 0$. If $\theta_{s}(t)=\frac{\theta(t / s)}{s}$ is correct, then $\varphi(t)=\frac{\partial \theta(t)}{\partial t}$ is defined as wavelet functions $\left(\theta_{s}(t)\right.$ signifies that $\theta(t)$ flexes under the scale factor $S$ ).

On the foundation of $\varphi(t)$ wavelet basis, the wavelet transform of signal $f(t)$ is:

$W_{f} f(t)=f(t) * \varphi(t)=s \frac{d\left[f(t) * \theta_{s}(t)\right]}{d t}$

Formula (3) shows the smoothing effect to the signal caused by $f(t) * \theta_{s}(t) . W_{f} f(t)$ is proportional to the first derivative of $f(t) * \theta_{s}(t)$ for different scales $S$. The relationship among the functions as $f(t), f(t) * \theta_{s}(t)$ and $W_{f} f(t)$ is displayed in Fig. (1).

The figure above expresses the singular points of signal $f(t)$ get through the $f(t) * \theta_{s}(t)$ inflexion which the wavelet corresponds to, $W_{f} f(t)$ manifests maximum values. Thus, solving the modulus maxima points of $W_{f} f(t)$ is equivalent to confirming the singular position of signal.

\subsubsection{Calculation of the Signal Singularity Degree}

The singularity degree of signal $f(x)$ at a certain point is commonly elaborated by the singularity index Lipschitz $\alpha$ [4], for short is Lip index. 


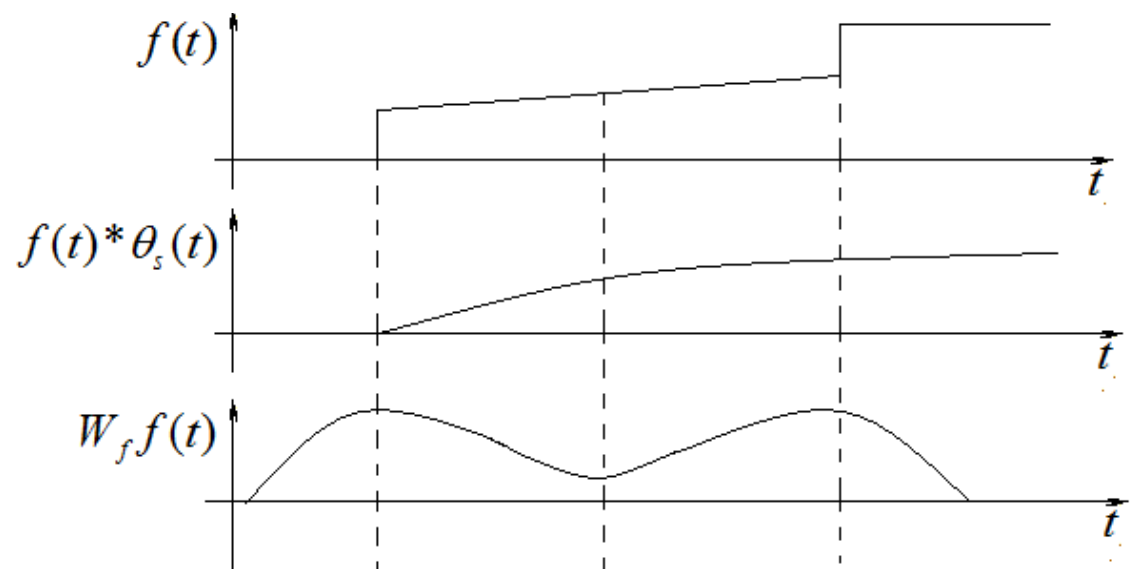

Fig. (1). Relationship between signal singularity points and wavelet modulus maxima values.

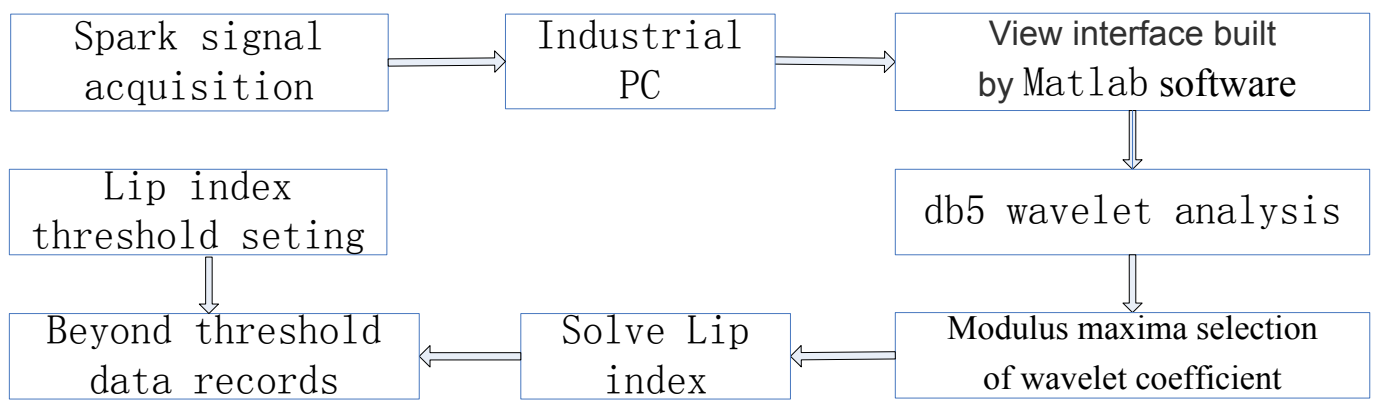

Fig. (2). Detection technology route of motor ring fire.

The definition of Lip index: assuming $0 \leq \alpha \leq 1$, a constant $\mathrm{K}$, in the $x_{0}$ point, makes the following formula fit among the $\mathrm{x}$ interval belonging to the neighborhood of $x_{0}$.

$$
\left|f(x)-f\left(x_{0}\right)\right| \leq K\left|x-x_{0}\right|
$$

Then $f(x)$ is called Lip $\alpha$ at $x_{0}$ point. Lip index $\alpha$ depicts the mutation degree of signal. And the greater or the smaller the $\alpha$ is, the greater or the smaller the signal singularity will be. Whereas the signal has no singularity at this place if $\alpha=1$ is correct.

The calculation formula of Lip index $\alpha$ is:

$$
\alpha=\log _{2} \frac{W_{f}\left(s_{i+1}, x_{0}\right)}{W_{f}\left(s_{i}, x_{0}\right)}
$$

$W_{f}\left(s_{i+1}, x_{0}\right)$ and $W_{f}\left(s_{i}, x_{0}\right)$ are respectively wavelet modulus maxima at the scales of $i$ and $i+1$.

\section{THE BASIC IDEA OF ELECTRIC MOTOR RING FIRE DETECTION}

This paper changes spark signal which is in motor commutator to electric signal that is easily handled through the ultraviolet photoelectric sensor, and applies the electric signal with amplification, noise reduction and A/D converter. It is converted to digital signal which will be sent to the console PC. In the end, the analysis of the spark signal is performed, combined with Matlab and wavelet analysis.

Detection system technical route is shown in Fig. (2).

\section{INSTANCE ANALYSIS}

Numerous electric sparks will be generated in motor commutator when the ring fire failure occurs. The verdict, from spectrum of electric spark, electric spark's principal energies concentrate upon $304 \mathrm{~nm}$ to $398 \mathrm{~nm}$ namely ultraviolet waveband. Hence, choosing the ultraviolet sensor may obtain superior acquisition sensitivity [5]. This paper utilizes ultraviolet photoelectric sensor to capture electric spark in commutator. It is the ring fire failure detection for the YQ1633 asynchronous traction motor of HXD1B type locomotive from a certain locomotive depot that is completed (Fig. 3). And the original signal is shown as Fig. (4).

\subsection{The Analysis of Wavelet Signal}

Db5 wavelet function is applied to conduct 6 layers decomposition of the signal. The layers from 1 to 6 of the high frequency components are received. And layers comprising 1, 3 and 6 are displayed in Fig. (5). 


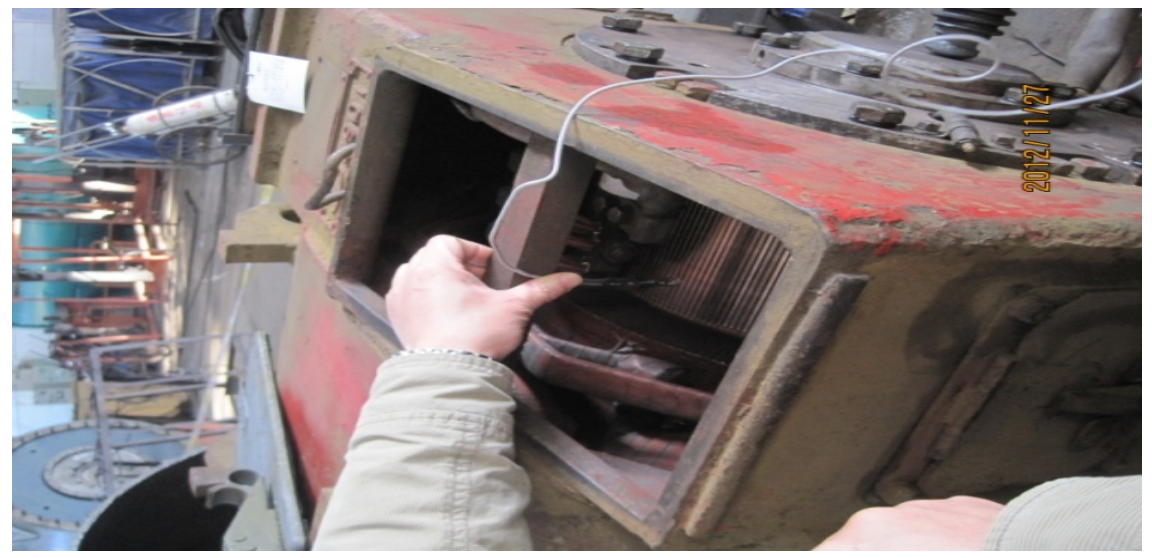

Fig. (3). Motor spark signal collection.

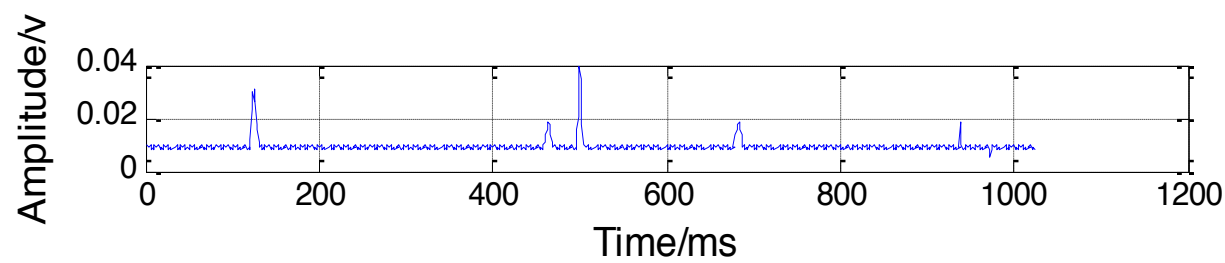

Fig. (4). Locomotive spark signals (Relationship of changing voltage amplitude gathered from ultraviolet photoelectric sensor with altering time).
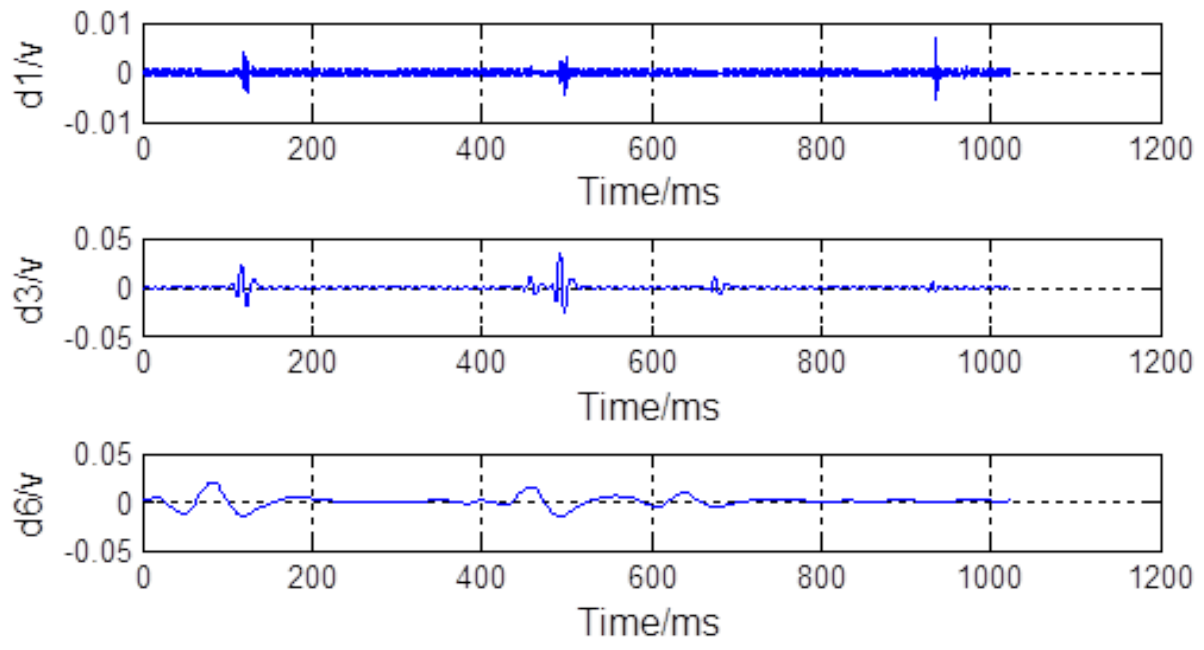

Fig. (5). High frequency detailed signals.

Fig. (5) the waveform of detailed signal components, obtained from wavelet decomposition. It can be discerned that the signal mutation probably occurred in both $110 \mathrm{~ms} \sim 150 \mathrm{~ms}$ and $480 \mathrm{~ms} \sim 500 \mathrm{~ms}$. Moreover, the mutation points of fault signal are revealed more and more clearly after 6 layers decomposition of the signal is completed.

\subsection{The Modulus Maxima Analysis of Wavelet Trans- forms}

It involves importing obtained signal to MATLAB software and using wave peak function to get the modulus maxima sequences of wavelet transform. Wave peak function is as below:

[signal,swa,swd,ddw,wpeak] = wave_peak(points,level,Lo_D,Hi_D,Lo_R,Hi_R,offset)

Among it: signal: original signal; swa: the low frequency information of wavelet; swd: the high frequency information of wavelet; ddw: local maximum position; wpeak: local maximum sequence; points: the length of processing data; level: the level of decomposition; wf: wavelet function name; [Lo_D,Hi_D,Lo_R,Hi_R]=wfilters(wf). 

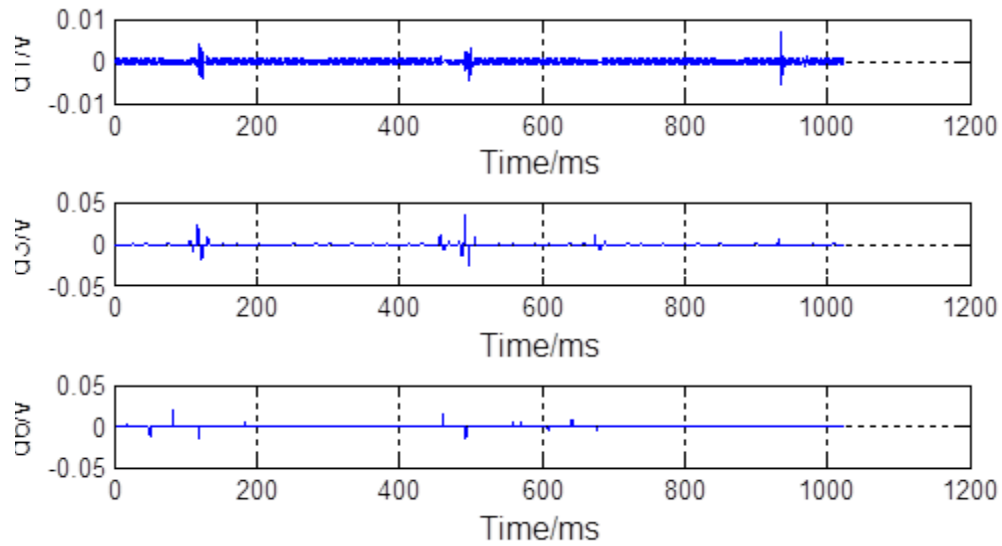

Fig. (6). Coefficient modulus maxima.

Modulus maxima of various scales are extracted by using wave peak function. Layers of 1, 3 and 6 are seen in Fig. (6).

Fig. (6) clearly shows the corresponding location and the size of signal modulus maxima.

By observing Figs. (1, 4 and $\mathbf{6})$, it can be observed that each scale of wavelet coefficient modulus maxima points corresponds to the location of the singular points of the original signal.

According to the different characteristics of modulus maxima between the noise and useful signal, the modulus maxima points whose amplitude decreases with the increasing scale are deleted, but the points whose amplitude increases with the magnifying scale are retained. At last, the positions of signal singular points with which these modulus maxima points correspond to are found [6].

\subsection{Lip Index Calculation}

A series of Lip index can be received by putting modulus maxima into the formula (5) of Lip index, and by comparing it with the grade criteria of motor spark signal, a proper Lip index threshold is selected. Then the Lip index will be obtained as follows, Lip index of 0.5342 in $120 \mathrm{~ms}$ and Lip 0.2538 index in $502 \mathrm{~ms}$. Through contrasting with the original signal, it is concluded that signal mutation degree is a little large when it is $502 \mathrm{~ms}$ and $120 \mathrm{~ms}$; and the degree of mutation with $502 \mathrm{~ms}$ is larger than with $120 \mathrm{~ms}$. It is consistent with the diagnosis of this paper, according to the principle that the smaller the Lip index is, the larger the singularity degree will be.

\section{CONCLUSION}

Through the wavelet analysis of the collected spark signal and according to the principle that the multi- scale modulus maxima points can locate the singularity of signal, the time of motor ring fire can be accurately judged. At the same time, the size of the traction motor spark can be determined after figuring out the signal singularity degree by means of modulus maxima. As a result, a new method for early diagnosis and inspection of the motor ring fire is provided.

\section{CONFLICT OF INTEREST}

The authors confirm that this article content has no conflict of interest.

\section{ACKNOWLEDGEMENTS}

This work was sponsored by the Jiangxi Province Postgraduate Students Special Funds for Innovation, and it was also funded by the Jiangxi Province Undergraduate Education Plan Foundation for Innovation \& Startups.

\section{REFERENCES}

[1] J. Xie, "The analysis and measures of motor ring fire causes on SS7 type electric locomotive traction motor", Elect. Drive Locomotives, vol. 3, pp. 101-102, 2013.

[2] C. Hu, G. Li, and T. Zhou, "System analysis and design of wavelet analysis based on MATLAB7.X", Xidian University Press, 2008.

[3] Q. Jiang, X. Lian, and X. Ye, "The selection of wavelet base function on structure damage detection", Logistics Technol., vol. 41, pp. 12-14, 2011

[4] X. Li and T. Qiao, "Singularity detection based on wavelet transform modulus maximum in magnetic memory signal of steel-cord belt", Coal Mine Mach., vol. 34, pp. 89-92, 2013.

[5] J. Liu, and W. Yu, "Study on monitoring device for looping spark of mining traction motors", Mining Mach., vol. 40, pp. 102-104, 2012.

[6] K. Lian, H. Wang, and B. Long, "A novel method on measuring Lipschitz exponent based on wavelet transform modulus maxima", Chin. J. Electron., vol. 36, pp. 107-110, 2008. 